\title{
Una aproximación al funcionamiento de los arroyos de montaña del norte de la Patagonia Andina
}

\author{
Verónica Díaz-Villanueva ${ }^{1, \bigotimes}$ \& Ricardo Albariño ${ }^{2}$ \\ ${ }^{1}$ Laboratorio de Limnología, INIBIOMA, Universidad Nacional del Comahue - CONICET. San Carlos de Bariloche, \\ Río Negro, Argentina. ${ }^{2}$ Laboratorio de Fotobiología, INIBIOMA, Universidad Nacional del Comahue - CONICET. San Carlos \\ de Bariloche, Río Negro, Argentina.
}

Resumen. Los Andes patagónicos tienen como vegetación característica los bosques de Nothofagus, que se extienden desde los $37^{\circ}$ a los $55^{\circ} \mathrm{S}$. En particular, N. pumilio (lenga) es la especie más ampliamente distribuida tanto en la Argentina como en Chile, y por ser decidua representa un notable pulso estacional de materia orgánica a los arroyos de cabecera. Estos arroyos tienen su origen en las alturas de las montañas y drenan las laderas hacia aguas bajas. Hace más de una década que se realizan estudios en la cuenca del Challhuaco, ubicada en la zona norte y en el extremo este de los Andes patagónicos. Los estudios abarcaron tanto aspectos estructurales como funcionales de la ecología de arroyos e incluyen el análisis de nutrientes y materia orgánica a lo largo de la cuenca, y relaciones alimentarias entre los diferentes componentes de sus tramas tróficas, desde productores primarios, descomponedores de detrito vegetal e invertebrados bentónicos hasta consumidores superiores como la trucha arcoíris. El objetivo de esta revisión fue integrar los resultados obtenidos sobre el funcionamiento de los sistemas fluviales de cabecera andino-patagónicos y su respuesta a factores de cambio espaciales (a lo largo de una cuenca) y temporales (entre estaciones). Finalmente, se destacan los conflictos y las amenazas más importantes que enfrentan estos sistemas (modificaciones en la cobertura vegetal, introducción de ganado, cambio climático) para señalar los aspectos que se deberían incorporar a las políticas de conservación, incluyendo un manejo sustentable de las actividades forestales y la cría de ganado.

[Palabras clave: arroyos de cabecera, arroyos andino patagónicos, Nothofagus pumilio, Parque Nacional Nahuel Huapi, cuenca del Challhuaco]

\begin{abstract}
An approach to the functioning of mountain streams in Northern Andean Patagonia. Patagonian Andes has Nothofagus forests as characteristic vegetation, which extends from $37^{\circ}$ to $55^{\circ} \mathrm{S}$. In particular, $N$. pumilio (lenga) is the most widely distributed species, both in Argentina and Chile, and as it is a deciduous species, it represents an important organic matter input to headwater streams. These streams are originated in high altitude mountains and drain the forest downstream. We have been studying the ecology of Challhuaco catchment, in the Northeast of Patagonian Andes, for more than a decade. These studies encompassed both structural and functional aspects of stream ecology, including nutrient and organic matter analysis along the catchment, and feeding relationship between the different components of food webs, from primary producers, leaf litter decomposers and benthic macroinvertebrates to top consumers, as the rainbow trout. In this review we aimed to gather and relate results of a catchment that may be used as a model to extrapolate to other Andean Patagonian catchments. Finally, we indicated some conflicts and threats to which these systems are exposed, to point out the aspects that should be incorporated into conservation policies including a more sustainable management of forestry and cattle raising.
\end{abstract}

[Keywords: headwater streams, Andean Patagonian streams, Nothofagus pumilio, Nahuel Huapi National Park, Challhuaco catchment]

Editor asociado: Esteban Jobbágy
Recibido: 28 de Octubre de 2020

Aceptado: 23 de Noviembre de 2020 


\section{INTRODUCCIÓN}

Los sistemas fluviales son elementos clave del paisaje. Se sitúan en la parte más baja del terreno y son las vías de drenaje del agua que cae a la superficie de la cuenca, la cual intercepta y drena el agua atmosférica hacia un mismo punto de salida. Por estas condiciones tienen una fuerte relación y dependencia con los eventos naturales y antrópicos que ocurren en el ecosistema terrestre circundante (Battin et al. 2008; Benstead and Leigh 2012). Otra de sus particularidades es que el agua se encuentra en movimiento siguiendo la pendiente del terreno y determina, a través de su recorrido y conjunción de canales de igual o diferente tamaño, un sistema complejo de forma más o menos dendrítica denominado red fluvial.

La región andino-patagónica está caracterizada por la presencia de bosques de Nothofagus (Cabrera and Willink 1980). Estos bosques proveen de sombreado y materia orgánica $(\mathrm{MO})$ alóctona a los arroyos de cabecera. La mayor extensión la ocupa el bosque caducifolio, representado por la lenga, N. pumilio (Poepp. et Endl) Krasser. Esta especie constituye el cinturón superior de bosque templado de montaña (entre 1000 y $1400 \mathrm{~m}$ s. n. m. en las latitudes menores) en los Andes del norte de la Patagonia, que alcanza la línea superior de vegetación (HildebrandVogel et al. 1990). Por debajo de los $1000 \mathrm{~m}$ de altitud dominan el coihue (Nothofagus dombeyi [Mirb.] Blume) y el ciprés de la cordillera (Austrocedrus chilensis [D. Don] Pic. Serm. and Bizzarri), ambas especies perennifolias. En esta zona de las cuencas, la influencia del dosel disminuye debido al aumento en el ancho de los arroyos, lo cual permite una mayor entrada de luz y, por ende, la condición de heterotrofía de sus cabeceras cambia a condiciones más autotróficas (Vannote et al. 1980; Xenopoulos et al. 2017). El balance entre los ingresos de energía alóctonos y autóctonos en los arroyos controlan la estructura de las distintas comunidades (Cummins and Klug 1979). Por lo tanto, la composición de la fauna bentónica de invertebrados reflejará las condiciones tróficas del sistema.

La mayoría de los estudios de arroyos de cabecera en la región patagónica abordan aspectos específicos de la estructura y funcionamiento, enfocados en un único compartimiento o en la interacción entre dos compartimientos bióticos. Por ejemplo, se han realizado numerosos estudios enfocados en los macroinvertebrados, sobre todo en la provincia de Chubut y los resultados se usaron para evaluar los diversos impactos de origen antrópico a los que están sometidos los ecosistemas fluviales (i.e., forestaciones de pino, introducción de ganado y urbanización) (Miserendino et al. 2008; Miserendino and Masi 2010; Miserendino et al. 2011; Miserendino et al. 2016). Los trabajos realizados en la provincia de Tierra del Fuego también se enfocaron en la biodiversidad de macroinvertebrados en sus cuatro ecorregiones (Villatarco Vazquez et al. 2019), y utilizaron a los macroinvertebrados como indicadores del impacto de las castoreras en los arroyos (Anderson et al. 2014; Anderson et al. 2020) y como indicadores de impacto antrópico (Zagarola et al. 2017; Anderson et al. 2018). El efecto de las castoreras también fue estudiado en las comunidades de autótrofos (fitoplancton y perifiton) (García and Rodríguez 2018; Rodríguez et al. 2020). Otro compartimiento biótico de interés ha sido la comunidad de consumidores tope, los peces, mayormente analizando la distribución y la coexistencia de las especies nativas y exóticas, y los efectos positivos o negativos de las especies exóticas sobre los ecosistemas (e.g., Buria et al. 2007; Aigo et al. 2008; Macchi et al. 2008; Di Prinzio and Casaux 2012).

En esta revisión sintetizamos y relacionamos los resultados obtenidos en numerosos estudios realizados en una cuenca del Parque Nacional Nahuel Huapi (PNNH). Estos estudios abarcan diversos componentes bióticos y abióticos y, en muchos casos, incluyen manipulaciones experimentales a escala de tramo. El objetivo es integrar los conocimientos sobre diferentes aspectos del funcionamiento de los sistemas fluviales andino-patagónicos y los factores de cambios que los alteran con especial énfasis en sus componentes bióticos. Se seleccionó la cuenca del Challhuaco como modelo por la cantidad de estudios efectuados (Tabla 1), que abarcan tanto aspectos hidrológicos, físico-químicos, como biológicos. Hemos organizado la información según el nivel trófico, describiendo las características y la dinámica de la materia orgánica de origen alóctono (detrito vegetal terrestre y materia orgánica disuelta) y autóctono (productores primarios), su utilización por parte de microorganismos descomponedores, la estructura de la comunidad de los consumidores primarios (mayormente macroinvertebrados) y el efecto de los peces 
Tabla 1. Título de los trabajos publicados y tesis de licenciaturas y doctorales realizados en la cuenca del Challhuaco.

Table 1. Title of published papers and thesis carried out in Challhuaco catchment.

\begin{tabular}{lll}
\hline Tema (título del trabajo) & Autores & Año \\
\hline Variables hidrológicas y físico-químicas & &
\end{tabular}

Efecto a largo plazo de los incendios forestales en la calidad del agua Temporetti, $\mathrm{P}$. de dos arroyos en la sub-región Andino-Patagónica, Argentina

Leaf litter dynamics in a forested small Andean catchment, northern Albariño, R., V. Díaz Villanueva, Patagonia, Argentina and L. Buria

Seasonal patterns of organic matter stoichiometry along a mountain Díaz Villanueva, V., M. Bastidas catchment.

Navarro, and R. Albariño

Caracterización de arroyos de cabecera temporarios y permanentes Mariluan, G. D. del norte de la patagonia andina

\section{Materia orgánica a lo largo de la cuenca}

Leaf litter dynamics in a forested small Andean catchment, northern Albariño, R., V. Díaz Villanueva, Patagonia, Argentina and L. Buria

Seasonal patterns of organic matter stoichiometry along a mountain Díaz Villanueva, V., M. Bastidas catchment.

Navarro, and R. Albariño

\section{Uso heterotrófico de la materia orgánica}

Leaf litter dynamics in a forested small Andean catchment, northern Albariño, R., V. Díaz Villanueva, Patagonia, Argentina and L. Buria

Leaf litter breakdown and benthic invertebrate colonization affected Mariluan, G. D., V. D. Villanueva, by seasonal drought in headwater lotic systems of Andean Patagonia and R. J. Albariño

High phosphorus content in leachates of the austral beech Nothofagus Bastidas Navarro, M., V. Díaz pumilio stimulates bacterioplankton C-consumption Villanueva, and B. Modenutti

Dissolved organic matter as P source for biofilms in two contrasting Díaz Villanueva, V. low-order streams

\section{Productores primarios}

Primary consumers and resources: Annual variation in two contrasting Díaz Villanueva, V., L. Buria, and R. 2010 reaches of a Patagonian mountain stream Albariño

Dissolved organic matter as P source for biofilms in two contrasting Díaz Villanueva, V.

\section{Consumidores primarios} andino.

Análisis de la fracción macrozoobentónica en un cuerpo lótico Albariño

Larval diet of the frog Alsodes gargola (Leptodactylidae: Telmatobiinae) Baffico, G. and C. Úbeda and some ecological considerations on its role in alpine and mountain aquatic environments in Patagonia.

Primary consumers and resources: Annual variation in two contrasting Díaz Villanueva, V., L. Buria, and R. 2010 reaches of a Patagonian mountain stream Albariño

Leaf litter breakdown and benthic invertebrate colonization affected Mariluan, G. D., V. D. Villanueva, by seasonal drought in headwater lotic systems of Andean Patagonia and R. J. Albariñ́o

Assessing the performance of macroinvertebrate metrics in the Mauad, M., M. L. Miserendino, M. Challhuaco-Ñireco System (Northern Patagonia, Argentina).

A. Risso, and J. Massaferro. 2015.

Caracterización de arroyos de cabecera temporarios y permanentes Mariluan, G. D. del norte de la patagonia andina

Disturbance disrupts the relation between community similarity and environmental distance at small spatial scale

\section{Depredadores}

Impact of exotic rainbow trout on the benthic macroinvertebrate community from Andean-Patagonian headwater streams.

Díaz Villanueva, V., G. Mariluan, and R. Albarino

Efecto de la depredación en la estructuración comunitaria del zoobentos Buria, L. en ambientes lóticos norpatagónicos

Does predation by the introduced rainbow trout cascade down to detritus and algae in a forested small stream in Patagonia?

Buria, L., R. Albariño, V. Díaz Villanueva, B. Modenutti, and E. Balseiro

Altered mayfly distribution due to strong interactions with alien Albariño, R. J. and L. M. Buria rainbow trout in Andean streams of Patagonia.

Evaluación de los costos y beneficios de la remoción de salmónidos Montañez, J. C. en un arroyo de montaña del Parque Nacional Nahuel Huapi como herramienta de manejo para la conservación.

Plantillas de hábitat y estructura de los ensambles de peces de arroyos Lallement, M. E. tributarios a la cuenca del río Limay Superior, Patagonia Argentina. 
como depredadores tope. Proponemos a) analizar, dentro de cada compartimento, los factores de cambio espaciales a lo largo de la cuenca, como el tamaño de los arroyos, el tipo de vegetación ribereña, la presencia de peces; b) analizar patrones temporales, sobre todo estacionales, y comparar estos resultados con los hallados en cuencas cercanas y en arroyos andino-patagónicos de otras latitudes. Muchos de los estudios analizados recurren a manipulaciones de variables ecológicas in situ, que permiten conocer la magnitud y dirección del cambio asociado a la variable controlada. Sin embargo, la escasa replicación de estudios experimentales de campo en este tipo de ambientes limita fuertemente comparaciones con otros sistemas fluviales de la región patagónica, y c) identificar y discutir los principales conflictos y amenazas que enfrentan o podrían enfrentar estos sistemas, para señalar los aspectos que deberían ser incorporados a las políticas de manejo y conservación de estos ecosistemas.

\section{La cuenca del Challhuaco}

Esta cuenca se encuentra en el límite este del bosque templado de Nothofagus, dentro del PNNH (Figura 1). Representa una condición de bosque en su extremo más seco, lo que sumado a la orientación norte determina un régimen hidrológico de menores precipitaciones (instantáneas y acumuladas) que en el oeste y estiajes más fuertes relacionados con la mayor evapotranspiración de laderas expuestas a insolación elevada. Abarca $47 \mathrm{~km}^{2}$, con sus nacientes a $1800 \mathrm{~m} \mathrm{s.} \mathrm{n.} \mathrm{m.,} \mathrm{y} \mathrm{drena} \mathrm{sus}$ aguas al arroyo Nireco, a 950 m s. n. m., el cual desemboca en el lago Nahuel Huapi. La vegetación del sotoboque de lenga está dominada por Berberis serrato-dentata Lechl., Myoschilos oblongum Ruiz and Pav., Ribes magellanicum Poir. y grandes extensiones de Alstroemeria aurea Graham.

La parte inferior de la cuenca presenta un fuerte impacto debido a un incendio que se produjo en enero de 1996 y que afectó alrededor de $4 \mathrm{~km}^{2}$ de bosque subalpino en el valle medio, con diferentes intensidades y extensiones entre subcuencas (Kitzberger et al. 2005). En el sector más bajo de la cuenca, la vegetación aún no se recuperó luego de más de veinte años del incendio y está dominada por N. antarctica, Diostea juncea (Gillies and Hook.) Miers, Schinus patagonica (Phil.) I.M. Johnst. y Vicia nigricans Hook. and Arn., entre otras (Mermoz et al. 2005) y especies exóticas como Pinus spp., Rosa rubiginosa L. (rosa mosqueta), Cytisus scoparycus (L.) Link (retama) y Cirsium vulgaris (Savi) Ten. (cardo negro) como las invasoras dominantes. El impacto humano hasta hace unos años estaba restringido al senderismo y la erosión provocada por el camino de acceso al bosque (hasta $\sim 1300 \mathrm{~m} \mathrm{~s}$. n. m.). Sin embargo, en los últimos 10 años ha aumentado considerablemente la extracción de leña y el pastoreo de ganado equino y vacuno y de ciervo colorado.

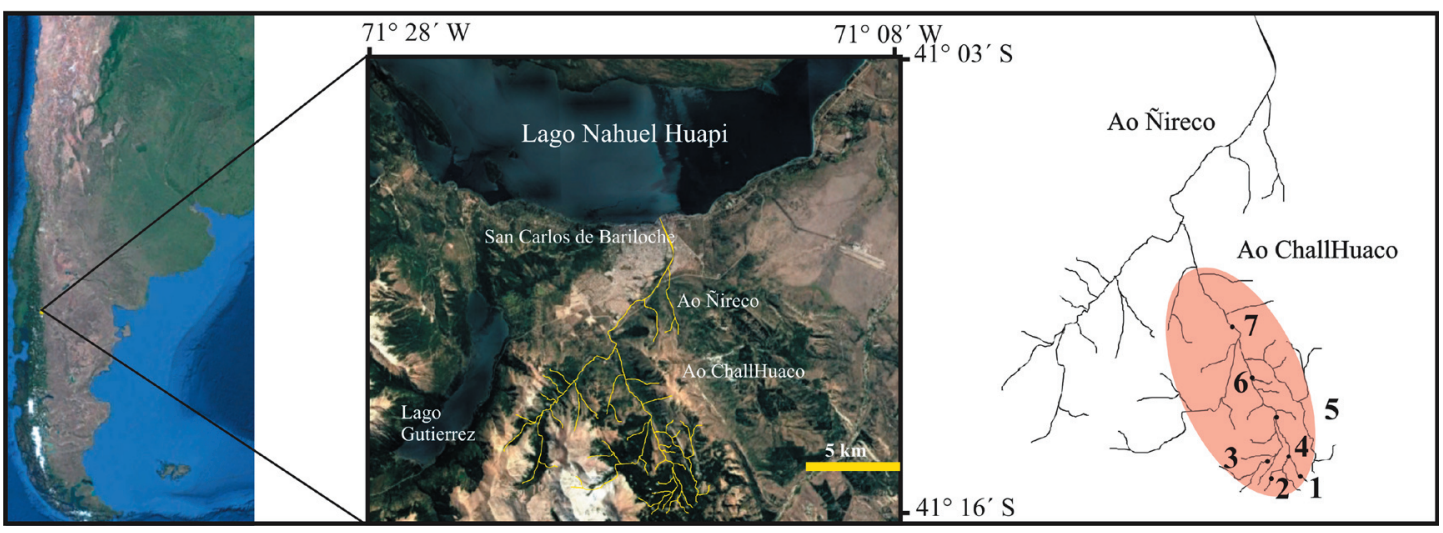

Figura 1. Ubicación geográfica de la cuenca del Challhuaco. Imagen satelital del área de estudio tomada de Google Earth, donde se marca la cuenca de los arroyos Challhuaco y Ñireco, y su desembocadura en el lago Nahuel Huapi luego de atravesar la ciudad de San Carlos de Bariloche, y detalle de las subcuencas, donde se marcan el área con los arroyos de cabecera donde se realizaron la mayoría de los estudios mencionados.

Figure 1. Geographic location of Challhuaco catchment. Satellite image of the study area taken from Google Earth, showing Challhuaco and Nireco river catchment and its outlet into Nahuel Huapi lake after running through San Carlos de Bariloche city, and detail of the sub-catchments, showing the area of headwater streams where most of the cited studies were carried out. 
Funcionamiento del ECOSISTEMA FLUVIAL

\section{A) Variables hidrológicas y físico-químicas}

Dos componentes fundamentales del funcionamiento de estos ecosistemas son las variables hidrológicas y físico-químicas, que están determinadas por factores regionales vinculados a la condición climática y geológica, y a factores locales como la orientación de la cuenca. Las cuencas del lago Nahuel Huapi drenan a través de basamentos ígneos estableciendo condiciones físicas y químicas homogéneas. Estos ambientes se caracterizan por aguas transparentes con concentración de oxígeno alta, temperaturas bajas y valores de $\mathrm{pH}$ neutros a ligeramente ácidos (Pedrozo et al. 1993). Además, son sistemas pobres en nutrientes, con una composición química dominada por calcio, bicarbonatos y sílice disuelto (Pedrozo et al. 1993). El clima en la región es templado frío, con temperaturas medias de $8.5^{\circ} \mathrm{C}$ y precipitaciones anuales que varían entre $2700 \mathrm{~mm}$ al oeste y 500 $\mathrm{mm}$ al este (Paruelo et al. 1998). En el arroyo Cahllhuaco, los valores de la temperatura del agua oscilan entre 0 y $4{ }^{\circ} \mathrm{C}$ durante el invierno, cuando pueden permanecer cubiertos por nieve, y entre $10^{\circ} \mathrm{C}$ (aguas arriba) y $14^{\circ} \mathrm{C}$ (aguas abajo) a fines del verano (marzo). La conductividad y la concentración de nutrientes inorgánicos son bajas, entre 30 y $93 \mu \mathrm{S} / \mathrm{cm}$ y con valores de fósforo reactivo soluble por debajo del límite de detección y hasta $8 \mu \mathrm{g}$ $\mathrm{P} / \mathrm{L}$, de amonio entre 1 y $15 \mu \mathrm{gN}-\mathrm{NH}_{4} / \mathrm{L}$ y de nitrato entre 2 y $85 \mu \mathrm{gN}-\mathrm{NO}_{3} / \mathrm{L}$ (Buria et al. 2007; Díaz Villanueva et al. 2010, 2018). Sin embargo, luego del incendio de 1996 se registraron valores de nitrato de hasta 1200 $\mu \mathrm{gN} / \mathrm{L}$ (Temporetti 2006), mientras los valores de los demás nutrientes se encontraban dentro de los rangos mencionados.

La morfología de los arroyos también influye en los procesos ecológicos (Elosegi and Sabater 2013). El cauce de los arroyos andinos es predominantemente escalonado en terrenos pronunciados, con frecuentes cascadas formadas por cortes verticales en la roca madre o bien generadas por la caída de troncos. Esta morfología determina la capacidad del sistema para retener detrito y también impide el acceso de los peces a los tramos superiores de la cuenca. Los cauces presentan alternancia de dos hábitats dominantes, pozones y rabiones, cuyas características condicionan a los ensambles de macroinvertebrados
(Montañez 2014; Mariluan 2017). La rugosidad del lecho en sus cabeceras está determinada por bloques, cantos rodados y guijarros y una gran abundancia de troncos y ramas que tienen una fuerte influencia física sobre el cauce y que favorecen a la acumulación de grava y arena en las zonas de deposición. Aguas abajo, a consecuencia de la disminución en su pendiente, el arroyo tiende a acumular mayor cantidad de sustratos finos (material aluvial) que provienen de la erosión agua arriba (Albariño et al. 2009).

En cuanto a las variables hidrológicas, los ecosistemas fluviales están sometidos a dos fuertes disturbios asociados al régimen hidrológico: las crecidas, asociadas al incremento marcado en sus caudales, y las sequías, con caudales marcadamente bajos pudiendo registrar períodos de caudal 'cero' (Poff et al. 1997); Gordon et al. 2004). Estos eventos pueden variar espacial y temporalmente en su magnitud, frecuencia, regularidad, duración y tasa de cambio (Kennard et al. 2010). De acuerdo con las características climáticas de la región patagónica, las precipitaciones son más abundantes entre otoño y primavera. Esta condición determina que los ríos y arroyos presenten hidrogramas bimodales con valores de máximo caudal, uno entre junio y julio, debido a las lluvias, y otro entre octubre y diciembre, debido al derretimiento de la nieve en sus cabeceras (Figura 2a). Durante el verano, el caudal disminuye gradualmente hasta mediados del otoño, determinando condiciones de estiaje definidas por caudales mínimos en los segmentos principales y secundarios y la interrupción del flujo de agua superficial en aquellos tributarios menores (Mariluan 2017) (Figura 2b). La sequía estacional representa el disturbio más común en los sistemas de cabecera, ya que estos arroyos son los que poseen los caudales más bajos de la cuenca (Gordon et al. 2004). En uno de los arroyos estudiados durante tres años (2011-2013), el régimen hidrológico mostró una extensión media anual sin agua superficial de 133 días ( 4 meses) (Figura $2 b$ ) (Mariluan 2017).

\section{B) Materia orgánica a lo largo de la cuenca}

Las cabeceras que se hallan por encima de la línea de árboles (1600 m s. n. m.) presentan condiciones de irradiancia alta y cobertura vegetal nula, lo cual sugiere que los ecosistemas están sustentados por 
a)
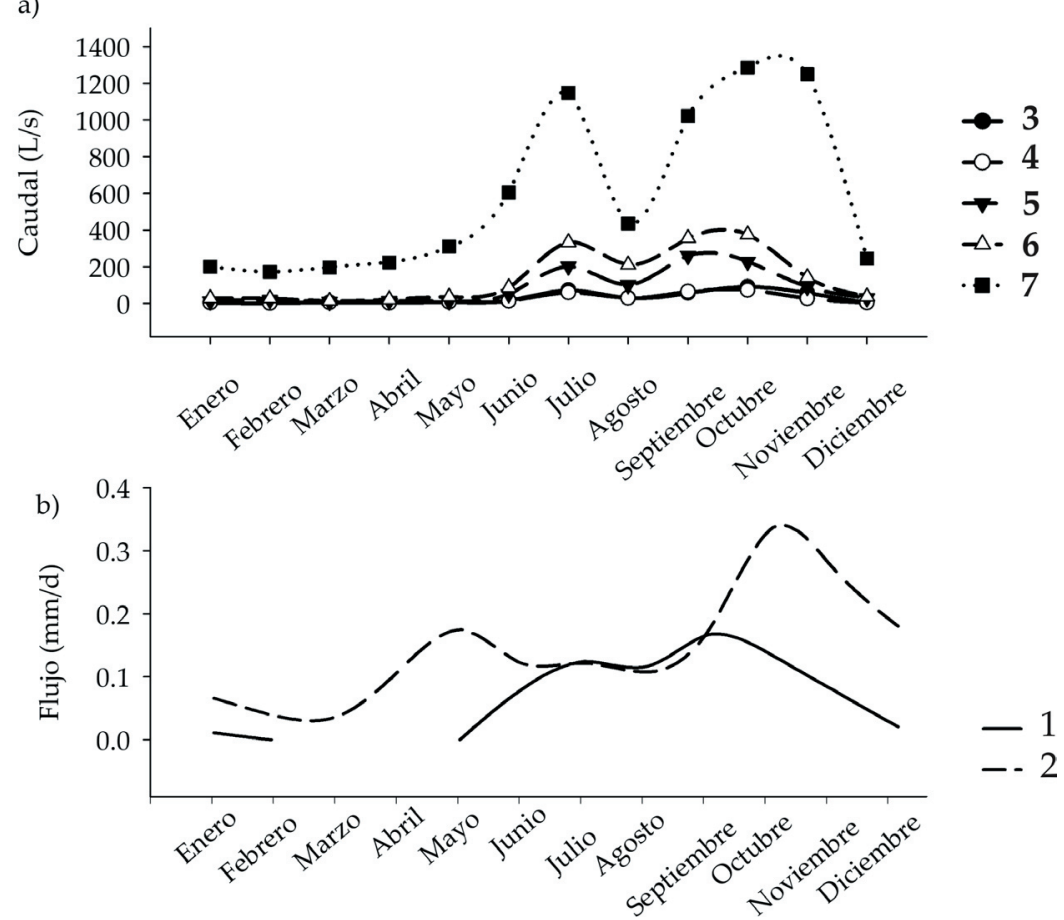

Figura 2. Variaciones temporales en el caudal en arroyos de diferente tamaño (orden) a lo largo de la cuenca del Challhuaco (año 2011). a) Caudales instantáneos (L/s) calculados mediante mediciones de la velocidad de corriente (con flujómetro Global Water, California, EEUU) y la sección del cauce, mensualmente durante un año, en cinco sitios (ver símbolos); b) Medias mensuales del flujo diario (mm/día) medidos de manera continua (con registradores HOBO U20001 Onset Co. complementados con medidas instantáneas de caudal mediante el método del trazador inerte (Gordon et al. 2004) en dos arroyos de orden 1, uno de régimen permanente (1) y otro de régimen temporario (2) y corregidos por superficie de la cuenca. La interrupción de la curva en el arroyo temporario indica flujo cero.

Figure 2. Temporal variations of discharge in streams of different sizes (stream order) along Challhuaco catchment (year 2011). a) Discharge (L/s) was obtained by measuring water velocity (with a water flow meter, Global Water, California, USA) and stream section monthly at five sites (see symbols); b) Mean monthly of daily fluxes (mm/day) measured continuously (with HOBO U20-001 Onset Co. dataloggers, supplemented with measurements of instantaneous discharge using the inert tracer method) (Gordon et al. 2004) in one perennial (1) and one temporary (2) first order streams, corrected by catchment area. Line interruption in T stream indicates cero flow.

los productores primarios, aun cuando la fotosíntesis se deprima debido a la fuerte insolación (Martyniuk et al. 2016). Dentro del bosque se producen aportes muy abundantes de materia orgánica vegetal a los arroyos, que incluyen hojarasca, material leñoso (escamas, ramas y troncos), flores y frutos. En particular, los arroyos de cabecera están cubiertos por el bosque de lenga, una especie caducifolia. Esta característica distingue al funcionamiento de estos arroyos de aquellos en donde las especies dominantes son perennes, como, por ejemplo, los bosques de coníferas. En los primeros, la hojarasca ingresa al sistema fundamentalmente durante otoño, mientras que en los últimos, el ingreso ocurre durante el verano (Richardson et al. 2005). En arroyos de bajo orden de la cuenca del Challhuaco, el material vegetal caído al lecho en 12 meses fue $423 \mathrm{~g} / \mathrm{m}^{2}$, de los cuales casi la mitad (200 $\mathrm{g} / \mathrm{m}^{2}$ ) ingresaron durante el mes de junio. El
$66 \%$ del material estuvo representado por hojas, de las cuales el 99\% fueron de lenga (Albariño et al. 2009) (los valores sólo incluyen las categorías de troncos y ramas menores a 5 $\mathrm{cm}$ de diámetro). Estas acumulaciones pueden tapizar gran parte del lecho fluvial al menos temporalmente, hasta el comienzo del ciclo hidrológico anual provocado por la estación de lluvias. Una fracción muy importante (entre 5 y $30 \mathrm{~g} / \mathrm{m}^{2}$ de hojarasca) (Albariño et al. 2009) permanece retenida por estructuras físicas del lecho como troncos, ramas y rocas (bloques y cantos rodados) que actúan de diques, o bien en hábitats deposicionales (con flujo bajo a despreciable), como los pozones. Este fenómeno es clave para que la hojarasca retenida sea procesada por microorganismos e invertebrados detritívoros.

Otra fracción de la hojarasca que ingresa a los arroyos es arrastrada aguas abajo y 
aporta recurso trófico para la comunidad de detritívoros que vive en los segmentos inferiores del ecosistema fluvial. La fracción de hojarasca exportada hacia abajo varía anualmente según la magnitud de las primeras precipitaciones de otoño. En un evento de lluvias intensas a comienzos del otoño de 2006 se registró un pulso de exportación que transportó $5 \mathrm{~g} / \mathrm{L}$ de hojas de lenga recién ingresadas al sistema acuático ( $\mathrm{km}$ aguas debajo de su origen. Aun así, el efecto que tiene el bosque sobre los arroyos que lo atraviesan determinó que la cantidad de hojarasca acumulada en el lecho fuera $30 \%$ mayor respecto de las extensiones fluviales inferiores en la cuenca (Albariño et al. 2009), lo cual revela la importancia del aporte de hojarasca desde las cabeceras hacia los tramos inferiores.

En cuanto a la materia orgánica particulada fina (MOPF) en transporte y la materia orgánica disuelta (MOD), su concentración y origen varían a lo largo de la cuenca (Díaz Villanueva et al. 2016). Tanto la concentración de MOPF como de MOD presentan una estacionalidad elevada, relacionada con la fenología del bosque y con el caudal. Una de las fuentes importantes de MOD son los lixiviados de la hojarasca (Tate and Meyer 1983; Meyer et al. 1998). Por lo tanto, en otoño, cuando se produce el mayor ingreso de $\mathrm{MO}$ durante la senescencia foliar en el bosque de lenga, se hallaron mayores concentraciones de MOD en las cabeceras y mayores valores de aromaticidad. Sin embargo, la disminución de la aromaticidad en los tramos inferiores de la cuenca fue menor a la esperada, teniendo en cuenta que allí debería dominar una mayor producción de derivados algales de baja aromaticidad (Bertilsson and Jones 2003). Esto indicaría que aguas abajo existen otros ingresos de MOD de alta aromaticidad que no necesariamente derivan de los lixiviados de hojas. En una cuenca cercana (arroyo Casa de Piedra) se demostró que aguas abajo, en el bosque perenne de coihue, la concentración de MOD sólo se relacionaba con los aumentos de caudal debido a las precipitaciones (Garcia et al. 2015) y que las propiedades ópticas del agua del arroyo (en invierno) eran más similares a los lixiviados de suelo, con alta aromaticidad, que a los lixiviados de hojas (Garcia et al. 2018). Por lo tanto, aguas abajo los lixiviados de hojas representan una fracción menor dentro del conjunto de la MOD.
Las relaciones estequiométricas (C:N:P) de la MOD halladas a lo largo de la cuenca del Challhuaco también fueron mayores (i.e., menor cantidad de nutrientes orgánicos) aguas arriba durante el otoño, demostrando que la MOD es más rica en nutrientes en los tramos inferiores, más abiertos, de la cuenca (Díaz Villanueva et al. 2016). Sin embargo, en la primavera y el verano hubo una disminución del C:N en los sitios agua arriba debido sobre todo a un aumento del N orgánico. Los ingresos de nitrógeno orgánico a los arroyos se atribuyeron al derretimiento de la nieve durante la primavera (Petrone et al. 2007).

\section{C) Uso heterotrófico de la materia orgánica}

La hojarasca retenida en el lecho comienza un proceso de colonización por microorganismos (i.e., hongos y bacterias) que acondicionan a la materia orgánica para ser consumida fundamentalmente por larvas de insectos mediante el proceso de fragmentación. Las tasas de descomposición microbiana de la lenga, medidas in situ, variaron entre $0.2 \mathrm{y}$ 0.5\%/día(otoño-inviernoeinvierno-primavera, respectivamente) mientras que al sumarles el efecto de los invertebrados detritívoros fueron de 0.6 y 0.7\%/día (Albariño et al. 2009; Mariluan et al. 2015). Por un lado, esto muestra que el reciclado es más rápido en primavera debido a la mayor temperatura del agua, y por el otro, que tanto los microorganismos descomponedores como los invertebrados detritívoros son claves en el proceso. Al compararlas con las tasas de descomposición de otras especies vegetales, las de lenga se encuentra entre las de tasa intermedia a baja según Petersen y Cummins (1974). Estas tasas de descomposición corresponden a arroyos cuyo régimen hídrico es permanente. Sin embargo, en la cuenca existen arroyos pequeños que, debido a su bajo caudal, se secan durante 2 a 4 meses. En estos arroyos de régimen temporario la tasa de descomposición debido al efecto conjunto de invertebrados y microorganismos es similar a la producida sólo por microorganismos $(0.3 \%$ /día, ambos casos), lo que sugiere que durante el otoño, cuando el ciclo hidrológico se restaura y al mismo tiempo cuando se produce la mayor caída de hojarasca, los invertebrados aún no han recolonizado los arroyos adecuadamente como para ejercer un efecto significativo sobre la descomposición de la hojarasca (Mariluan et al. 2015). 
En cuanto a la MOD, no sólo provee C, sino también nutrientes (e.g., $\mathrm{P}$ y N) a la comunidad microbiana. La MOD liberada por la hojarasca (lixiviados) de lenga contiene una alta concentración de P (Díaz Villanueva et al. 2016). En un estudio experimental realizado con la comunidad bacteriana de una laguna de altura en la cuenca de Challhuaco (laguna Los Patos) conectada a la red fluvial, se demostró que el $\mathrm{P}$ aportado por los lixiviados de lenga promueve el consumo de MOD (Bastidas Navarro et al. 2019). En los arroyos, la mayor parte del reciclado de nutrientes sucede en el biofilm, consorcio formado por microorganismos autótrofos y heterótrofos adheridos a un sustrato sólido (Bernhardt and Likens 2002; Ylla et al. 2010). La comparación de los nutrientes y las capacidades metabólicas de biofilms de dos arroyos de segundo orden en la cuenca del Challhuaco, muy cercanos y similares pero con diferentes concentraciones de MOD, mostró que el biofilm del arroyo con mayor concentración de MOD no sólo tenía mayor biomasa y concentración de clorofila 'a' durante todo el año, sino que también contenía mayor cantidad de P (Díaz Villanueva 2019). Además el biofilm del arroyo con mayor concentración de MOD tuvo mayor capacidad para utilizar P orgánico (glucosa1-P). Por el contrario, el biofilm proveniente del arroyo con menor concentración de MOD utilizó preferentemente moléculas nitrogenadas (aminoácidos y aminas). La utilización de nitrógeno orgánico por biofilms que crecen en condiciones de bajas concentraciones de carbono coincide con resultados experimentales hallados por Ghosh y Leff (2013). Estos resultados revelaron que la biomasa tanto heterotrófica como autotrófica de los biofilms está relacionada con la concentración de MOD y que el uso de los nutrientes orgánicos también puede estar relacionado con la cantidad de MOD en el ecosistema.

\section{D) Productores primarios}

En los arroyos, la mayor biomasa de productores primarios se desarrolla sobre la superficie del fondo (e.g., piedras, arena, etc.), en la matriz del biofilm. En los arroyos de bosque, la biomasa de los productores primarios está muy limitada por la cantidad de luz que reciben. En el arroyo Challhuaco, la intensidad de luz fotosintéticamente activa (LFA) en las cabeceras boscosas (medida en un día diáfano) puede variar entre 200 y 300 $\mu \mathrm{E} / \mathrm{m}^{2}$ en otoño y en verano, respectivamente, mientras que aguas abajo, donde el arroyo corre por un valle de vegetación abierta, la intensidad varía entre $600 \mu \mathrm{E} / \mathrm{m}^{2}$ en otoño y $2000 \mu \mathrm{E} / \mathrm{m}^{2}$ en verano (Díaz Villanueva et al. 2010). Sin embargo, al comparar dos sitios cercanos, uno adentro del bosque de lenga y otro en un sitio abierto, la biomasa de autótrofos en el biofilm fue mayor en el bosque (Díaz Villanueva et al. 2010). Estas diferencias pueden deberse a que la irradiancia en el sitio abierto es alta y puede ejercer foto-inhibición. En arroyos cercanos a la cuenca del Challhuaco, Martyniuk et al. (2016) demostraron que las diferencias en irradiancia causan diferencias en las eficiencias fotosintéticas, con valores óptimos cuando la LFA oscila entre los 300 y $750 \mu \mathrm{E} / \mathrm{m}^{2}$. Estas diferencias en la irradiancia entre el bosque y los sectores abiertos llevan a mayores relaciones entre el $\mathrm{C}$ y la clorofila, con mayor fijación de $\mathrm{C}$ por unidad de biomasa (menor contenido de clorofila) en los sitios de mayor intensidad lumínica. Otra diferencia, además de la biomasa, es que en los arroyos de bosque dominan las Bacillariophyta (diatomeas), que pueden alcanzar el 100\% de la diversidad, mientras que en sitios abiertos pasa a dominar durante la primavera la Crysophyta filamentosa Hydrurus foetidus (Buria et al. 2010; Díaz Villanueva et al. 2010), que florece durante el derretimiento de nieve y en condiciones de caudal elevado.

\section{E) Consumidores primarios}

Como en muchos otros ecosistemas, la comunidad de invertebrados, caracterizada en los arroyos de montaña por especies adaptadas a la vida en el fondo, representa la principal conexión entre los recursos basales del ecosistema, sean detrito o productores primarios, y los componentes superiores de la trama trófica (Cummins and Klug 1979). La fauna de invertebrados bentónicos de la cuenca del Challhuaco está dominada por estadíos larvales de insectos terrestres de cinco órdenes, Diptera, Coleoptera, Ephemeroptera, Plecoptera y Trichoptera, y de oligoquetos, con presencia también del platelminto Romankenkius patagonicus (Buria et al. 2007; Montañez 2014; Mauad et al. 2015; Mariluan 2017; Díaz Villanueva et al. 2018). Tanto los arroyos de cabecera como a lo largo de la cuenca del Challhuaco (de menor a mayor orden) presentan una composición taxonómica espacio-temporal homogénea de sus ensambles, con abundancia de dípteros de las familias Chironomidae, Simulidae y Tipulidae, efemerópteros de las familias 
Baetidae, Leptophlebiidae y Nesameletidae, plecópteros de las familias Austroperlidae, Gripopterygidae y Notonemouridae y tricópteros de las familias Hydrobiosidae, Hydropsychidae, Limnephilidae y Sericostomatidae. Esta composición a nivel de familia y géneros coincide mayormente con la que se encuentra en arroyos andinopatagónicos de cabecera a latitudes mayores (Miserendino 2001).

Una distinción con respecto de las comunidades de invertebrados que caracterizan a arroyos a menor altitud (incluyendo a la cuenca inferior del Challhuaco en su desagüe al lago Nahuel Huapi, Arroyo Nireco) (Figura 1) es la ausencia de dos grupos de invertebrados: los crustáceos omnívoros del género Aegla y los moluscos raspadores del género Chilina (Albarino 1994; Mauad et al. 2015). Por otra parte, los arroyos del Challhuaco menores a $2 \mathrm{~m}$ de ancho también son habitados por estadíos larvales del anfibio Alsodes gargola, la rana de palmada de arroyo. Los renacuajos viven en los pozones durante todo el año, alimentándose de MOPF depositada y de perifiton (Baffico and Úbeda 2006). Estos vertebrados son un componente abundante y probablemente tengan un papel clave junto a los invertebrados en el reciclado de la materia orgánica y los nutrientes, materia que aún no ha sido abordada. La diversidad de anfibios es incrementada por la presencia de mallines que permanecen inundados en invierno y primavera y están conectados a la red fluvial. En estos sistemas habitan otras especies de anfibios (Pleurodema thaul, Pleurodema bufoninum y Rhinella spinulosa), que son depredados por larvas de Odonata, Notonectidae y Dytiscidae (Jara and Perotti 2010).

Entre los arroyos de cabecera existen diferencias en la diversidad de invertebrados, que fueron atribuidas en parte a la duración del ciclo hídrico. Mientras que en los arroyos permanentes más pequeños hay un predominio de larvas de efemerópteros, seguida por tricópteros y dípteros, en los temporarios co-dominan las larvas de dípteros Chironomidae y los oligoquetos. Sin embargo, fuera de la excepción del hallazgo de poblaciones del tricóptero Verger capillatus (Trichoptera), exclusivo en los arroyos temporarios (Mariluan 2017), estos ecosistemas no presentaron una fauna única, sino más bien un subconjunto de las especies presentes en los arroyos permanentes. Esto sugiere que varias especies tienen estrategias de resistencia (soportar la sequía in situ) o resiliencia (recolonizar desde otros hábitats o ecosistemas que no se secan) ante la sequía estacional que sufren los arroyos de cabecera. Probablemente ambas estrategias coexistan ya que son comunes en otros ecosistemas fluviales temporarios (Leigh 2012; Stubbington and Datry 2013).

La similitud entre las comunidades de invertebrados en arroyos de una misma cuenca o de cuencas cercanas depende tanto de la capacidad de dispersión de las especies que conforman la comunidad (Clarke et al. 2008; Altermatt et al. 2013) como de los gradientes ambientales a lo largo de la cuenca (Mykrä et al. 2007; Heino and Mykrä 2008). A esta complejidad se le agrega la distancia entre sitios, la topología de la red fluvial $\mathrm{y}$, especialmente, el efecto que tienen los disturbios ecológicos en la diversidad, los cuales pueden desencadenar efectos opuestos. Por un lado, un disturbio puede crear nuevas oportunidades para las especies colonizadoras, aumentando la diversidad (Hutchinson 1953; Townsend and Scarsbrook 1997; Hawkins et al. 2015), mientras que, por otro lado, puede excluir especies que no posean las adaptaciones necesarias para las nuevas condiciones, disminuyendo la diversidad (Death 2002). El incendio ocurrido en 1996 (ver más arriba) que resultó en una baja tasa de recuperación (Mermoz et al. 2005) determinó diferencias en la cobertura vegetal variando con la intensidad del fuego. Las consecuencias del incendio se expresaron en cambios en la comunidad de invertebrados dependiendo de su intensidad y extensión en las subcuencas mientras la cercanía entre arroyos no sirvió como fuente de colonización desde los arroyos sin fuego a los quemados (Díaz Villanueva et al. 2018). A pesar de no hallar una relación inversa entre las distancias geográficas o ambientales y la similitud de las comunidades, al aumentar la escala espacial del análisis incorporando datos de los ensambles bentónicos de otros sitios de la cuenca, incluyendo sitios del arroyo Ñireco (Mauad et al. 2015), se halló que la similitud en los ensambles era explicada por ambas distancias independientemente (ambiental y geográfica). Estos resultados enfatizan la influencia de un disturbio de magnitud de escala de paisaje (cuenca) en la vegetación ribereña sobre las comunidades bentónicas cuyas consecuencias persisten a 10 años del evento.

Los arroyos del Challhuaco que atraviesan el bosque de lenga se caracterizan por 
una comunidad dominada en densidad y biomasa por invertebrados detritívoros que consumen mayormente detrito proveniente de la vegetación ribereña (Albariño et al. 2009; Díaz Villanueva et al. 2010; Mauad et al. 2015). El disturbio sobre la vegetación ribereña se reflejó sobre la composición taxonómica de los invertebrados del bentos mediados por los cambios en su diversidad funcional. La disminución de la cobertura vegetal provocó una disminución de la proporción de fragmentadores, a favor de los raspadores (Figura 3), principalmente los raspadores, especializados en alimentarse del biofilm (Díaz Villanueva et al. 2018). Este efecto diferencial de los cambios en la cobertura vegetal ribereña sobre la composición funcional en arroyos también había sido registrado en una comparación de los invertebrados de diferentes tramos del mismo arroyo, aguas arriba en un sitio de bosque y aguas abajo en un sitio de vegetación abierta o matorral (Díaz Villanueva et al. 2010). Los resultados confirmaron la relación entre la abundancia de los diferentes grupos funcionales de alimentación con la abundancia de los recursos. En particular, el grupo de detritívoros fragmentadores dominaron en el sitio de bosque, mientras que en el sitio de pastizal-matorral se vieron favorecidos los herbívoros raspadores (Díaz Villanueva et al. 2010).

\section{F) Depredadores, completando la trama trófica}

El compartimiento superior de las tramas tróficas lo constituyen fundamentalmente los peces. También hay invertebrados depredadores de tamaño pequeño y mediano que probablemente tienen importancia en la mortalidad de los estadíos más tempranos de desarrollo de las especies de detritívoros y herbívoros de la comunidad (Modenutti et al. 2010). La depredación por parte de los adultos y juveniles de Alsodes gargola puede jugar cierto control sobre los invertebrados acuáticos ya que la dieta de otros Alsodes patagónicos (e.g., A. coppingeri en Chile) incluye a larvas de insectos acuáticos (Alveal et al. 2015).

Los depredadores pueden ejercer un control fuerte desde arriba sobre el resto de la trama trófica a través de la depredación directa sobre sus presas; inclusive, alterando el comportamiento de sus presas sólo por estar presentes en el ecosistema (detectables de manera visual o por sus excreciones) (Ferrari et al. 2010; Schäffer et al. 2013). Cuando este control desde arriba es fuerte, sus poblaciones pueden afectar propiedades ecológicas fundamentales de los ecosistemas, con un efecto directo sobre la composición, la abundancia y la producción secundaria de los ensambles de invertebrados, o indirecto sobre la composición y la abundancia de los productores primarios y su producción, la abundancia y la descomposición de la materia orgánica y el reciclado de nutrientes (Vitousek 1990; Peckarsky et al. 2013; Rodríguez-Lozano et al. 2016).

En la Patagonia, la introducción de salmónidos ha sido una práctica común desde principios del siglo XX. Hoy, la fauna de peces de la Patagonia está dominada principalmente por la trucha arcoíris (Oncorhynchus mykiss), la trucha marrón (Salmo trutta) y la trucha de arroyo (Salvelinus fontinalis) (Pascual et al. 2002; Vigliano and Alonso 2007). En la cuenca del Challhuaco, el ensamble de peces está exclusivamente caracterizado por los Salmonidae, del cual la trucha arcoíris representa el $94 \%$ y la trucha de arroyo el $6 \%$ restante (Lallement 2017). Su distribución está restringida a los segmentos inferiores por la existencia de barreras físicas a su dispersión hacia arriba (e.g., saltos que no pueden sortear) (Albariño et al. 2009). Estas barreras permiten realizar experimentos naturales, ya que reflejan el efecto del establecimiento de estos depredadores en el ecosistema al comparar tramos con y sin peces (Figura 3). La trucha arcoíris se alimenta principalmente de los invertebrados más vulnerables a la depredación, ya sea por su tamaño o por su movilidad (Buria et al. 2007; Buria 2008, Albariño and Buria 2011; Montañez 2014). De esta manera, este depredador tope estructura a la comunidad al eliminar o reducir la abundancia de las especies de mayor tamaño y de aquellas con un modo de vida que los exponga a la depredación visual; esto incluye también al anfibio $A$. gargola, que está ausente en los segmentos con truchas (Montañez 2014).

El efecto de la depredación por parte de los peces sobre los invertebrados se traduce en efectos indirectos (y opuestos) sobre los recursos que explotan los consumidores primarios (los invertebrados), es decir, sobre el perifiton y sobre la hojarasca (Buria et al. 2010). Si bien varios estudios han demostrado el efecto en cascada sobre la cadena trófica autotrófica, en la que el efecto de depredación sobre los herbívoros libera de la presión de pastoreo a los productores primarios (Bechara et al. 1992; Katano et al. 2003; Meissner and Muotka 2006), 

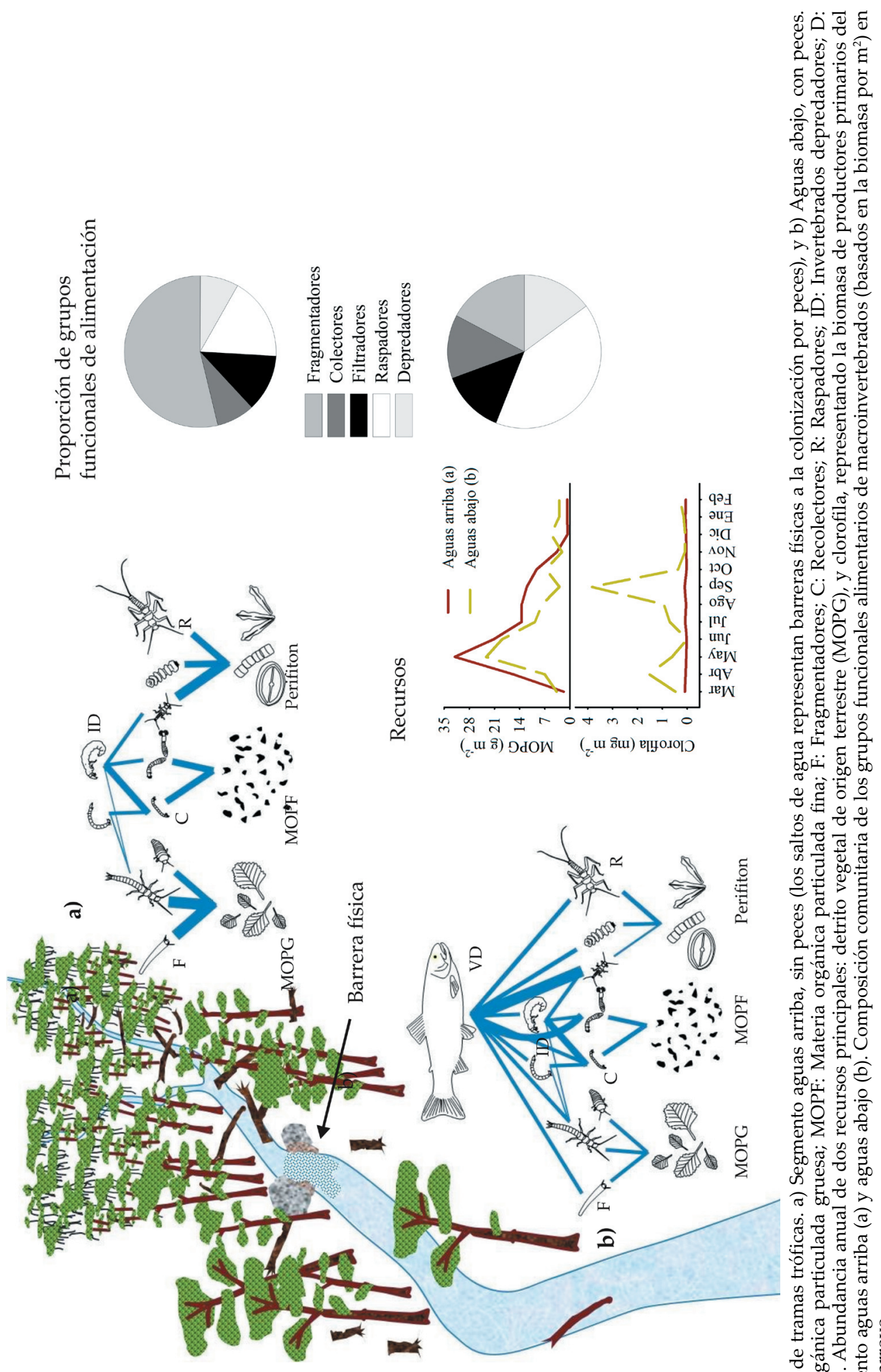

$\frac{\nabla}{\sigma} \ddot{g} \hat{e}$

可

卷

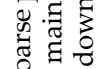

iํ.

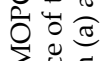

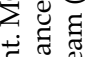

है

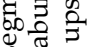

矛

医专

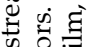

产造高苛

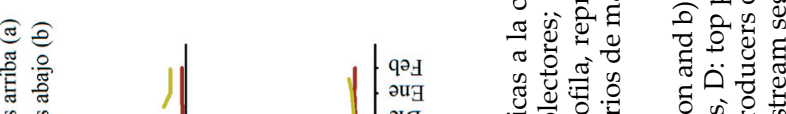

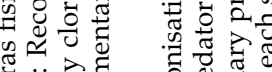

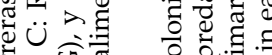

चै

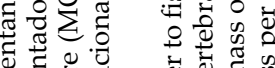

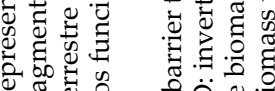

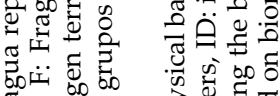

过

为

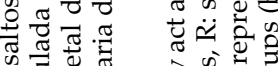

क

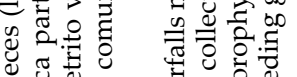

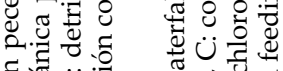

का

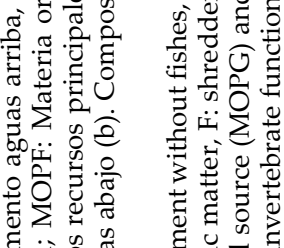

至灾各

ส

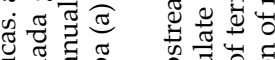

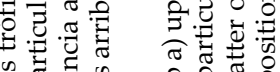

व

可

ช

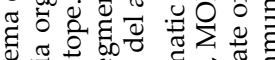

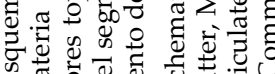

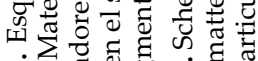

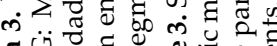

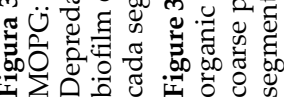


el efecto sobre la descomposición permanecía incierto. Buria et al. (2010) demostraron no sólo que el efecto de los herbívoros raspadores se reduce en presencia de truchas, resultando en un lecho con biomasa perifítica hasta 10 veces mayor que en los sitios sin truchas, sino también que la tasa de reciclado de hojarasca durante el verano fue menor en los sitios con truchas y se reflejó en $60 \%$ más de acumulación de hojarasca en el lecho.

\section{Alcances del análisis}

Los aspectos sintetizados hasta aquí han demostrado que el funcionamiento de los arroyos está muy ligado a la dinámica, la fenología y las características geológicas y bioquímicas del bosque dominante en la cuenca, y a las condiciones climáticas que gobiernan la región. Proponemos que nuestros resultados son extrapolables a otras redes fluviales de montaña con escaso o nulo impacto antrópico y cuya vegetación dominante en la cuenca es el bosque de lenga. Estas condiciones son mayormente comunes en toda la Patagonia andina, en especial porque existen semejantes condiciones climáticas y porque las cabeceras fluviales suelen encontrase en zonas alejadas de las actividades humanas. Aún queda mucho por dilucidar; por ejemplo, cuánto afecta la orientación dominante de una cuenca. Es posible que las cuencas orientadas al sur se distingan de las orientadas al norte en relación con las diferencias en el régimen térmico, de irradiancia y de evapotranspiración, en particular en las áreas más áridas, afectando a la biota y su intervención en los procesos ecológicos (Egli et al. 2009; Yetemen et al. 2015). Es probable que aquellas cuencas y redes similares, pero que atraviesan bosques mixtos con latifoliadas y coníferas perennes, determinen contrastes relevantes en la dinámica de la materia orgánica y la energía (Kominoski and Rosemond 2011; Guevara et al. 2018), lo cual condicionaría a las tramas tróficas acuáticas respecto de las de los bosques monoespecíficos de hojas caducas.

\section{Amenazas sobre los ARroyos}

Comprende el funcionamiento de un sistema en su estado prístino permite considerar las amenazas y los problemas a los que están sometidos, y ponderar la dirección y la intensidad de las alteraciones consecuentes. En particular, estas cuencas enfrentan diversas amenazas de origen antrópico y por cuestiones de síntesis abordamos aquellos aspectos/especies que son más relevantes. La urbanización es un fenómeno creciente en la región, sobre todo en cercanías del área de estudio (Figura 1). Este fenómeno se encuentra entre los que generan las alteraciones más drásticas sobre los ecosistemas terrestres y acuáticos (las cuencas). La ciudad de Bariloche, al colindar con el PNNH en la zona de estudio, genera presiones indirectas sobre la cuenca, vinculadas con la alteración del paisaje por remoción de vegetación (i.e., tala no autorizada), aumento del riesgo de incendios accidentales, introducción de especies exóticas invasoras y mascotas (APN 2018: Plan de Gestión del PNNH). Los característicos impactos de los ecosistemas urbanos (e.g., la alteración del régimen hidrológico y térmico del ecosistema acuático, enriquecimiento por nutrientes y aporte de sustancias tóxicas, ingreso de sedimentos, entre otros [Douglas and James 2015]), son despreciables sobre la cuenca de estudio sólo porque estos fenómenos se expresan siguiendo la pendiente del terreno $\mathrm{y}$, por lo tanto, afectan al ecosistema ya fuera del área protegida, aguas abajo de la cuenca estudiada.

\section{Cambios en la vegetación terrestre}

Los cambios en los ingresos de materia orgánica generan un impacto muy fuerte en las redes fluviales (Kominoski et al. 2011). Los bosques andino-patagónicos sufren principalmente dos tipos de alteraciones: los incendios y la introducción de especies. Los incendios, tanto naturales como intencionales (Veblen et al. 1992; Veblen et al. 2011) alteran por muchos años a los sistemas debido a la baja recuperación del bosque (Díaz Villanueva et al. 2018). Por otro lado, el uso de la madera del bosque con diferentes fines domésticos y comerciales por parte de comunidades rurales y urbanas que habitan dentro o están en contacto con áreas protegidas es una práctica común en la región (APN 2019; Plan de Gestión del PNNH). Estos factores pueden disminuir fuertemente el ingreso de material leñoso a los arroyos, con importantes consecuencias sobre el ecosistema fluvial. Los troncos cumplen una función clave en la morfología y la estructura de los arroyos al disminuir la velocidad de la corriente $\mathrm{y}$, sobre todo, al formar diques que retienen la materia orgánica y el sedimento inorgánico (Richardson et al. 2005; Mao et al. 2008; Gurnell and Grabowski 2016). Su ausencia aumenta la exportación de sedimentos y afecta el reciclado de nutrientes (Elosegi and Sabater 2013). 
En cuanto a la introducción de especies con uso comercial, como las Pináceas, si bien las grandes plantaciones con fines comerciales se hallan mayormente en la estepa, también existen forestaciones en la zona de transición y el bosque, incluyendo a la cuenca baja del Challhuaco. Estas especies son importantes invasoras cuando se escapan y dispersan naturalmente desde las forestaciones (Sarasola et al. 2006; Pauchard et al. 2015; Raffaele et al. 2015). Las forestaciones alteran la abundancia y la calidad del material vegetal aportado a los arroyos respecto de arroyos en bosques naturales (Albariño and Balseiro 2002; Balseiro and Albarino 2006; Albariño et al. 2009). En una forestación de $P$. ponderosa y $P$. radiata en Meliquina, Neuquén, la cantidad de detrito caído al arroyo durante mayo fue $450 \mathrm{~g} / \mathrm{m}^{2}(90 \%$ constituido por acículas), lo cual es 3.5 veces superior a la biomasa aportada a un arroyo en el bosque nativo (Albariño et al. 2009). Más cercano al Challhuaco, en un arroyo de cabecera del bosque de lenga en la cuenca del arroyo Nireco, la tasa de descomposición de acículas de $P$. ponderosa fue $50 \%$ más baja que la de la hojarasca de lenga $(0.2 \%$ /día vs. $0.3 \%$ / día, respectivamente) (Albariño and Balseiro 2002). Las características físicas y químicas de las acículas de estas coníferas contrastan con las de las hojas de especies latifoliadas nativas, lo cual determina su baja calidad como recurso para descomponedores y detritívoros (Albariño and Balseiro 2002; Balseiro and Albarino 2006). En los arroyos que atraviesan forestaciones de pinos en sitios más al sur de los Andes patagónicos, como así también en la provincia de Córdoba, se demostró que las comunidades de invertebrados acuáticos resultan muy alteradas (Miserendino and Masi 2010; Márquez et al. 2015) como consecuencia de la mala calidad de las acículas como recurso para los detritívoros respecto del detrito originado por la vegetación ribereña dominante (Albariño and Balseiro 2002; Márquez et al. 2017).

\section{Introducción de ganado}

La cría de ganado vacuno de subsistencia tiene una presencia histórica en el PNNH, con impactos negativos sobre el ecosistema terrestre (e.g., sobre la vegetación nativa y su tasa de reciclado) (Blackhall et al. 2008; Piazza et al. 2018; Ballari et al. 2020). En el norte de Chubut, la cría intensiva y semi-intensiva tiene efectos negativos en los arroyos de montaña y pedemontanos. Ambas prácticas afectan de forma notable la integridad ecológica de las riberas, el hábitat fluvial y la comunidad de invertebrados acuáticos (Miserendino et al. 2011; Horak et al. 2019). Por su parte, el impacto de la ganadería de subsistencia (la principal modalidad de cría dentro de los Parques Nacionales de Patagonia) y sus consecuencias sobre los sistemas acuáticos aún no fueron abordadas. Si bien la abundancia de ganado se redujo en los últimos años (cantidad total declarada por pobladores del PNNH en 2017: 700 unidades; APN 2019; Plan de Gestión del PNNH), las áreas donde se cría reciben fuertes impactos negativos. En el valle del Challhuaco, la presencia de ganado vacuno comenzó hace más de 10 años; las observaciones de campo muestran la alteración de la vegetación del sotobosque, con claros signos de ramoneo, incluyendo a los renovales de lenga. La presencia de caballos y de ciervo colorado incrementa la presión sobre estos ecosistemas. La erosión del suelo terrestre, de los mallines y del lecho de arroyos ha crecido de manera muy notoria en los últimos 10 años. Si bien las vacas usan las riberas y sus arroyos menos de $10 \%$ del tiempo de actividad, son propensas a defecar dentro o cerca de estos (Bond et al. 2012). Esta actividad tiene consecuencias inmediatas asociadas a los ingresos de sedimentos, nutrientes y patógenos a los arroyos, y es probable también que la fisonomía del bosque de lenga sea alterada, reflejándose a largo plazo en el funcionamiento fluvial (i.e., alteración del sombreado y del aporte de madera y hojarasca al arroyo).

\section{Cambio climático}

El reciente informe del Panel Intergubernamental para el Cambio Climático (IPCC 2018) sostiene que, aun cuando se logre desacelerar el calentamiento del planeta en las próximas décadas, los cambios en los regímenes de la temperatura y de las precipitaciones profundizarán el movimiento y la extinción de especies, así como el cambio en la composición de las comunidades y en el funcionamiento de los ecosistemas. Uno de los procesos clave en ecosistemas acuáticos que se verá muy afectado es la mineralización de la materia orgánica vegetal terrestre, controlada mayormente por dos factores: la humedad y la temperatura. Un experimento a escala global sobre el efecto de la temperatura, realizado en 22 arroyos distribuidos en todos los continentes, entre 0.4 y 48.0 grados de latitud (incluyendo un sitio en la cuenca del Challhuaco), mostró que la descomposición microbiana aumentó $0.2 \%$ por cada grado centígrado mientras que, por el contrario, 
la descomposición mediada por detritívoros disminuyó en una magnitud similar (Boyero et al. 2011). En consecuencia, el efecto neto de la temperatura sobre la mineralización del detrito vegetal fue nulo. Sin embargo, el hallazgo tiene implicancias en la biogeoquímica global, ya que la descomposición microbiana y la mediada por detritívoros representan diferentes vías y magnitudes de transformación de la materia orgánica en $\mathrm{CO}_{2}$, lo que determina un mayor flujo de ese gas hacia la atmósfera.

En cuanto al régimen de precipitaciones, los escenarios para la Patagonia anticipan sequías más frecuentes e intensas (Nuñez et al. 2009; Dai 2012), lo cual aumentaría la duración de la fase seca y la cantidad y extensión de arroyos con régimen intermitente. En el estudio de Mariluan et al. (2015), en el que se comparó la descomposición en un arroyo temporario contra uno permanente durante la primavera (i.e., en la fase con flujo de agua continuo), las tasas de descomposición resultaron similares. Sin embargo, la temperatura, registrada constantemente a lo largo de todo el experimento, fue en promedio $1^{\circ} \mathrm{C}$ mayor en el arroyo intermitente. Por lo tanto, cuando se compararon las tasas de descomposición eliminando el efecto de la temperatura, estas fueron 2 veces mayores en el arroyo permanente. Este resultado está en línea con el descripto arriba en el escenario previsto para la región, donde los flujos de $\mathrm{CO}_{2}$ aumentarán como consecuencia de una mayor tasa de descomposición, debido al efecto superpuesto de mayores temperaturas y mayor extensión y número de arroyos temporarios.

Finalmente, dos trabajos recientes en arroyos temporarios, realizados en 29 países entre los cuales se incluyeron arroyos temporarios de la cuenca del Challhuaco, demostraron que en el momento en que estos arroyos vuelven a tener agua se liberan grandes cantidades de $\mathrm{CO}_{2}$ tanto por descomposición de la hojarasca acumulada en el lecho seco (Datry et al. 2018) como desde los sedimentos (Von Schiller et al. 2019). En particular, se halló que los sedimentos con mayor cantidad de materia orgánica, con menores valores de C:N y mayor cobertura ribereña, en cuencas naturales y más húmedas, como las andino-patagónicas, eran las que liberaban mayores cantidades de $\mathrm{CO}_{2}$ a la atmósfera.

\section{Conclusiones}

Los arroyos de cabecera de los Andes patagónicos aún son mayormente ambientes prístinos que albergan una alta biodiversidad y que conservan el funcionamiento de su ecosistema inalterado, lo cual depende en gran medida del estado del bosque de lenga. Además de los aspectos ambientales, estos ecosistemas cumplen un rol trascendental porque en ellos se concentra la producción de agua de calidad para consumo humano, riego de cultivos y producción de energía hidroeléctrica (Pessacg et al. 2015). Sin embargo, los impactos antrópicos ya han alterado de manera considerable sistemas que se encuentran en las cercanías de áreas urbanas, como Esquel, Ushuaia y Bariloche, entre otras. Una característica del poblamiento humano en la Patagonia Andina es que se estableció en la zona de transición entre el bosque y la estepa. Esto determina una mayor presión humana sobre los ecosistemas acuáticos localizados en el límite más xérico de distribución del bosque, y una mayor vulnerabilidad, como nuestra cuenca de estudio. El bosque de lenga en esta zona presenta muy baja a nula tasa de regeneración y se lo considera un bosque viejo (Heinemann et al. 2000), relicto de un clima más húmedo. Dada la fuerte conectividad entre los ecosistemas terrestre y acuático, cada (in)decisión de manejo en el uso de la tierra es una decisión indirecta sobre el ecosistema acuático. Esto requiere poner una atención especial en su manejo para conservar el bosque y los sistemas acuáticos asociados. Conocer los sistemas en estado prístino permite establecer líneas de base con las cuales considerar la dirección y la magnitud de cambio ante forzantes antrópicos, y constituye una herramienta poderosa para identificar las amenazas y poder señalarlas a los entes responsables del manejo y cuidado de los recursos naturales.

Agradecimientos. Agradecemos a las instituciones que han hecho posible estos estudios. Agencia Nacional de Promoción Científica y Técnica, Proyectos PICT 20184385 y PICT 2016-0959. VDV y RA son investigadores de CONICET. También, a los revisores anónimos que aportaron al mejoramiento del manuscrito. 


\section{REFERENCIAS}

Aigo, J., V. Cussac, S. Peris, S. Ortubay, S. Gómez, H. López, M. Gross, J. Barriga, and M. Battini. 2008. Distribution of introduced and native fish in Patagonia (Argentina): patterns and changes in fish assemblages. Reviews in Fish Biology and Fisheries 18:387-408. https://doi.org/10.1007/s11160-007-9080-8.

Albariño, R. J. 1994. Análisis de la fracción macrozoobentónica en un cuerpo lótico andino. Universidad Nacional del Comahue, Centro Regional Universitario Bariloche.

Albariño, R., and E. Balseiro. 2002. Leaf litter breakdown in Patagonian streams: native versus exotic trees and the effect of invertebrate size. Aquatic Conservation: Marine and Freshwater Ecosystems 12:181-192. https://doi.org/ 10.1002/aqc.511.

Albariño, R., V. Díaz Villanueva, and L. Buria. 2009. Leaf litter dynamics in a forested small Andean catchment, northern Patagonia, Argentina. Pp. 183-211 in N. V. P. B. C. O. R. Godoy (ed.). Ecological Advances on Chilean temperate rainforests. Academia Press.

Albariño, R. J., and L. M. Buria. 2011. Altered mayfly distribution due to strong interactions with alien rainbow trout in Andean streams of Patagonia. Limnologica 41:220-227. https://doi.org/10.1016/j.limno.2010.07.004.

Altermatt, F., M. Seymour, N. Martínez, and J. Sadler. 2013. River network properties shape $\alpha$-diversity and community similarity patterns of aquatic insect communities across major drainage basins. Journal of Biogeography 40:2249-2260. https://doi.org/10.1111/jbi.12178.

Alveal, N., H. Díaz-Páez, A. Henríquez, and O. Vergara. 2015. Aspectos dietarios de Alsodes coppingeri Günther, 1881 (Anura: Alsodidae) en Chile. Gayana (Concepción) 79:5-10. https://doi.org/10.4067/S0717-65382015000100002.

Anderson, C. B., M. Johnson, and M. E. Lopez. 2018. Establishing habitat-specific indicator species in Tierra del Fuego with freshwater macroinvertebrates. New Zealand Journal of Marine and Freshwater Research 52:145-154. https: //doi.org/10.1080/00288330.2017.1329747.

Anderson, C. B., M. Tagliaferro, A. Fisk, A. D. Rosemond, M. L. Sánchez, and M. T. Arts. 2020. Fatty acids elucidate sub-Antarctic stream benthic food web dynamics invaded by the North American beaver (Castor canadensis). Polar Biology 43:423-433. https://doi.org/10.1007/s00300-020-02644-z.

Anderson, C. B., M. Vanessa Lencinas, P. K. Wallem, A. E. Valenzuela, M. P. Simanonok, and G. Martínez Pastur. 2014. Engineering by an invasive species alters landscape-level ecosystem function, but does not affect biodiversity in freshwater systems. Diversity and distributions 20:214-222. https://doi.org/10.1111/ddi.12147.

Baffico, G., and C. Úbeda. 2006. Larval diet of the frog Alsodes gargola (Leptodactylidae: Telmatobiinae) and some ecological considerations on its role in alpine and mountain aquatic environments in Patagonia. Amphibia-Reptilia 27:161-168. https://doi.org/10.1163/156853806777239986.

Balseiro, E., and R. J. Albarino. 2006. C-N mismatch in the leaf litter-shredder relationship of an Andean Patagonian stream detritivore. Journal of the North American Benthological Society 25:607-615. https://doi.org/10.1899/08873593(2006)25[607:CMITLL]2.0.CO;2.

Ballari, S. A., A. E. Valenzuela, and M. A. Nunez. 2020. Interactions between wild boar and cattle in Patagonian temperate forest: cattle impacts are worse when alone than with wild boar. Biological invasions 22:1681-1689. https: //doi.org/10.1007/s10530-020-02212-w.

Bastidas Navarro, M., V. Díaz Villanueva, and B. Modenutti. 2019. High phosphorus content in leachates of the austral beech Nothofagus pumilio stimulates bacterioplankton C-consumption. Freshwater Science 38(3):435-447. https: //doi.org/10.1086/704752.

Battin, T., S. Findlay, E. Marti, L. A. Kaplan, J. D. Newbold, C. S. Hopkinson, A. I. Packman, and F. Sabater. 2008. Biophysical controls on organic carbon fluxes in fluvial networks. Nature Geoscience 1:95-100. https://doi.org/ 10.1038/ngeo101.

Bechara, J. A., G. Moreau, and D. Planas. 1992. Top-down effects of brook trout (Salvelinus fontinalis) in a boreal forest stream. Canadian Journal of Fisheries and Aquatic Sciences 49:2093-2103. https://doi.org/10.1139/f92-233.

Benstead, J. P., and D. S. Leigh. 2012. An expanded role for river networks. Nature Geoscience 5(10):678-679. https: //doi.org/10.1038/ngeo1593.

Bernhardt, E. S., and G. Likens. 2002. Dissolved organic carbon enrichment alters nitrogen dynamics in a forest stream. Ecology 83:1689-1700. https://doi.org/10.1890/0012-9658(2002)083[1689:DOCEAN]2.0.CO;2.

Bertilsson, S., and J. B. Jones. 2003. Supply of dissolved organic matter to aquatic ecosystems: autochthonous sources. Pp. 3-24 in R. Findlay and R. L. Sinsabaugh (eds.). Aquatic Ecosystems. Elsevier. https://doi.org/10.1016/B978012256371-3/50002-0.

Blackhall, M., E. Raffaele, and T. T. Veblen. 2008. Cattle affect early post-fire regeneration in a Nothofagus dombeyiAustrocedrus chilensis mixed forest in northern Patagonia, Argentina. Biological Conservation 141:2251-2261. https: //doi.org/10.1016/j.biocon.2008.06.016.

Bond, T. A., D. Sear, and M. Edwards. 2012. Temperature-driven river utilisation and preferential defecation by cattle in an English chalk stream. Livestock Science 146:59-66. https://doi.org/10.1016/j.livsci.2012.02.022.

Boyero, L., R. G. Pearson, M. O. Gessner, L. A. Barmuta, V. Ferreira, M. A. Graca, D. Dudgeon, A. J. Boulton, M. Callisto, E. Chauvet, J. E. Helson, A. Bruder, R. J. Albarino, C. M. Yule, M. Arunachalam, J. N. Davies, R. Figueroa, A. S. Flecker, A. Ramirez, R. G. Death, T. Iwata, J. M. Mathooko, C. Mathuriau, J. F. Goncalves, Jr., M. S. Moretti, T. Jinggut, S. Lamothe, C. M'Erimba, L. Ratnarajah, M. H. Schindler, J. Castela, L. M. Buria, A. Cornejo, V. D. Villanueva, and D. C. West. 2011. A global experiment suggests climate warming will not accelerate litter decomposition in streams but might reduce carbon sequestration. Ecol Lett 14:289-294. https://doi.org/10.1111/j.1461-0248.2010.01578.x. 
Buria, L. 2008. Efecto de la depredación en la estructuración comunitaria del zoobentos en ambientes lóticos norpatagónicos. Tesis Doctoral: Centro Regional Universitario Bariloche, Universidad Nacional del Comahue.

Buria, L., R. Albariño, V. Díaz Villanueva, B. Modenutti, and E. Balseiro. 2010. Does predation by the introduced rainbow trout cascade down to detritus and algae in a forested small stream in Patagonia? Hydrobiologia 651:161-172. https: //doi.org/10.1007/s10750-010-0293-9.

Buria, L., R. Albariño, V. D. Villanueva, B. Modenutti, and E. Balseiro. 2007. Impact of exotic rainbow trout on the benthic macroinvertebrate community from Andean-Patagonian headwater streams. Fundamental and Applied Limnology / Archiv für Hydrobiologie 168:145-154. https://doi.org/10.1127/1863-9135/2007/0168-0145.

Cabrera, A., and A. Willink. 1980. Biogeografía de América Latina: Washington. Organización de Estados Americanos, serie Biología 13:122.

Clarke, A., R. Mac Nally, N. Bond, and P. S. Lake. 2008. Macroinvertebrate diversity in headwater streams: a review. Freshwater Biology 53:1707-1721. https://doi.org/10.1111/j.1365-2427.2008.02041.x.

Cummins, K. W., and M. J. Klug. 1979. Feeding ecology of stream invertebrates. Annual review of ecology and systematics 10:147-172. https://doi.org/10.1146/annurev.es.10.110179.001051.

Dai, A. 2012. Increasing drought under global warming in observations and models. Nature Climate Change 3:52-58. https://doi.org/10.1038/nclimate1633.

Datry, T., A. Foulquier, R. Corti, D. Von Schiller, K. Tockner, C. Mendoza-Lera, J.-C. Clement, M. Gessner, M. Moleón, and R. Stubbington. 2018. A global analysis of terrestrial plant litter dynamics in non-perennial waterways. Nature Geoscience 11:497-503. https://doi.org/10.1038/s41561-018-0172-y. https://doi.org/10.1038/s41561-018-0134-4.

Death, R. G. 2002. Predicting invertebrate diversity from disturbance regimes in forest streams. Oikos 97:18-30. https: //doi.org/10.1034/j.1600-0706.2002.970102.x.

Di Prinzio, C. Y., and R. J. Casaux. 2012. Dietary overlap among native and non-native fish in Patagonian low-order streams. Pp. 21-30 in Annales de Limnologie-International Journal of Limnology. EDP Sciences. https://doi.org/ 10.1051/limn/2011055.

Díaz Villanueva, V. 2019. Dissolved organic matter as P source for biofilms in two contrasting low-order streams. Fundamental and Applied Limnology/Archiv für Hydrobiologie 193:131-142. https://doi.org/10.1127/fal/2019/1234.

Díaz Villanueva, V., M. Bastidas Navarro, and R. Albariño. 2016. Seasonal patterns of organic matter stoichiometry along a mountain catchment. Hydrobiologia 771:227-238. https://doi.org/10.1007/s10750-015-2636-z.

Díaz Villanueva, V., L. Buria, and R. Albariño. 2010. Primary consumers and resources: Annual variation in two contrasting reaches of a Patagonian mountain stream. Annales de Limnologie - International Journal of Limnology 46:21-28. https://doi.org/10.1051/limn/2010003.

Díaz Villanueva, V., G. Mariluan, and R. Albarino. 2018. Disturbance disrupts the relation between community similarity and environmental distance at small spatial scale. Ecological Research 33:225-236. https://doi.org/10.1007/s11284017-1539-z.

Elosegi, A., and S. Sabater. 2013. Effects of hydromorphological impacts on river ecosystem functioning: a review and suggestions for assessing ecological impacts. Hydrobiologia 712:129-143. https://doi.org/10.1007/s10750-012-1226-6.

Ferrari, M. C., B. D. Wisenden, and D. P. Chivers. 2010. Chemical ecology of predator-prey interactions in aquatic ecosystems: a review and prospectus. Canadian Journal of Zoology 88:698-724. https://doi.org/10.1139/Z10-029.

García, R. D., M. d. C. Diéguez, M. Gerea, P. E. García, and M. Reissig. 2018. Characterisation and reactivity continuum of dissolved organic matter in forested headwater catchments of Andean Patagonia. Freshwater Biology 63:1049-1062. https://doi.org/10.1111/fwb.13114.

García, R. D., M. Reissig, C. P. Queimalinos, P. E. García, and M. C. Dieguez. 2015. Climate-driven terrestrial inputs in ultraoligotrophic mountain streams of Andean Patagonia revealed through chromophoric and fluorescent dissolved organic matter. Sci Total Environ 521-522:280-292. https://doi.org/10.1016/j.scitotenv.2015.03.102.

García, V. J., and P. Rodríguez. 2018. Beavers' presence affects metabolism of periphyton and limnological variables in Fuegian rivers and streams. Ecologia Austral 28:593-605. https://doi.org/10.25260/EA.18.28.3.0.708.

Ghosh, S., and L. G. Leff. 2013. Impacts of labile organic carbon concentration on organic and inorganic nitrogen utilization by a stream biofilm bacterial community. Applied and Environmental Microbiology 79:7130-7141. https: //doi.org/10.1128/AEM.01694-13.

Gordon, N. D., T. A. McMahon, and B. L. Finlayson. 2004. Stream hydrology: an introduction for ecologists. John Wiley and Sons.

Guevara, G., R. Godoy, and M. Franco. 2018. Linking riparian forest harvest to benthic macroinvertebrate communities in Andean headwater streams in southern Chile. Limnologica 68:105-114. https://doi.org/10.1016/j.limno.2017.07.007.

Gurnell, A. M., and R. C. Grabowski. 2016. Vegetation-hydrogeomorphology interactions in a low-energy, humanimpacted river. River Research and Applications 32:202-215. https://doi.org/10.1002/rra.2922.

Hawkins, C. P., H. Mykrä, J. Oksanen, and J. J. Vander Laan. 2015. Environmental disturbance can increase beta diversity of stream macroinvertebrate assemblages. Global Ecology and Biogeography 24:483-494. https://doi.org/ 10.1111/geb.12254.

Heinemann, K., T. Kitzberger, and T. T. Veblen. 2000. Influences of gap microheterogeneity on the regeneration of Nothofagus pumilio in a xeric old-growth forest of northwestern Patagonia, Argentina. Canadian Journal of Forest Research 30:25-31. https://doi.org/10.1139/x99-181. https://doi.org/10.1139/cjfr-30-1-25.

Heino, J., and H. Mykrä. 2008. Control of stream insect assemblages: roles of spatial configuration and local environmental factors. Ecological Entomology 33:614-622. https://doi.org/10.1111/j.1365-2311.2008.01012.x. 
Hildebrand-Vogel, R., R. Godoy, and A. Vogel. 1990. Subantarctic-andean Nothofagus pumilio forests. Vegetatio 89: 55-68. https://doi.org/10.1007/BF00134434.

Horak, C. N., Y. A. Assef, and M. L. Miserendino. 2019. Assessing effects of confined animal production systems on water quality, ecological integrity, and macroinvertebrates at small piedmont streams (Patagonia, Argentina). Agricultural water management 216:242-253. https://doi.org/10.1016/j.agwat.2019.01.026.

Hutchinson, G. E. 1953. The concept of pattern in ecology. Proceedings of the Academy of Natural Sciences of Philadelphia 105:1-12.

IPCC. 2018. Special report on the impacts of global warming of $1.5 \mathrm{C}$ above pre-industrial levels and related global greenhouse gas emission pathways, in the context of strengthening the global response to the threat of climate change, sustainable development, and efforts to eradicate poverty, in 2018: Global warming of 1.5 C, ed. V. World Meteorological Organization, Geneva, Switzerland.

Jara, F. G., and M. G. Perotti. 2010. Risk of predation and behavioural response in three anuran species: influence of tadpole size and predator type. Hydrobiologia 644:313-324. https://doi.org/10.1007/s10750-010-0196-9.

Katano, O., Y. Aonuma, T. Nakamura, and S. Yamamoto. 2003. Indirect contramensalism through trophic cascades between two omnivorous fishes. Ecology 84:1311-1323. https://doi.org/10.1890/0012-9658(2003)084[1311: ICTTCB]2.0.CO;2.

Kennard, M. J., B. J. Pusey, J. D. Olden, S. J. Mackay, J. L. Stein, and N. Marsh. 2010. Classification of natural flow regimes in Australia to support environmental flow management. Freshwater Biology 55:171-193. https://doi.org/ 10.1111/j.1365-2427.2009.02307.x.

Kitzberger, T., E. Raffaele, K. Heinemann, and M. J. Mazzarino. 2005. Effects of fire severity in a north Patagonian subalpine forest. Journal of Vegetation Science 16:5-12. https://doi.org/10.1111/j.1654-1103.2005.tb02333.x.

Kominoski, J. S., L. B. Marczak, and J. S. Richardson. 2011. Riparian forest composition affects stream litter decomposition despite similar microbial and invertebrate communities. Ecology 92:151-159. https://doi.org/10.1890/10-0028.1.

Kominoski, J. S., and A. D. Rosemond. 2011. Conservation from the bottom up: forecasting effects of global change on dynamics of organic matter and management needs for river networks. Freshwater Science 31:51-68. https://doi.org/ 10.1899/10-160.1.

Lallement, M. E. 2017. Plantillas de hábitat y estructura de los ensambles de peces de arroyos tributarios a la cuenca del río Limay Superior, Patagonia Argentina. Universidad Nacional del Comahue. URL: rdi.uncoma.edu.ar:8080/ handle/123456789/179.

Leigh, C. 2012. Dry-season changes in macroinvertebrate assemblages of highly seasonal rivers: responses to low flow, no flow and antecedent hydrology. Hydrobiologia 703:95-112. https://doi.org/10.1007/s10750-012-1347-y.

Macchi, P. J., P. H. Vigliano, M. Pascual, M. Alonso, M. A. Denegri, D. Milano, M. G. Asorey, and G. Lippolt. 2008. Historical policy goals for fish management in northern continental Patagonia Argentina: A structuring force of actual fish assemblages? Pp. 331 in American Fisheries Society Symposium. American Fisheries Society.

Mao, L., S. Burns, F. Comiti, A. Andreoli, A. Urciuolo, M. Gaviño-Novillo, R. Iturraspe, and M. Aristide Lenzi. 2008. Acumulaciones de detritos leñosos en un cauce de montaña de Tierra del Fuego: análisis de la movilidad y de los efectos hidromorfológicos. Bosque (Valdivia) 29:197-211. https://doi.org/10.4067/S0717-92002008000300003.

Mariluan, G. D. 2017. Caracterización de arroyos de cabecera temporarios y permanentes del norte de la patagonia andina. PhD. Universidad Nacional del Comahue.

Mariluan, G. D., V. D. Villanueva, and R. J. Albariño. 2015. Leaf litter breakdown and benthic invertebrate colonization affected by seasonal drought in headwater lotic systems of Andean Patagonia. Hydrobiologia 760:171-187. https: //doi.org/10.1007/s10750-015-2324-z.

Márquez, J. A., L. Cibils, R. E. Principe, and R. J. Albariño. 2015. Stream macroinvertebrate communities change with grassland afforestation in central Argentina. Limnologica 53:17-25. https://doi.org/10.1016/j.limno.2015.05.002.

Márquez, J. A., R. E. Principe, L. Cibils Martina, and R. J. Albariño. 2017. Pine needle litter acts as habitat but not as food source for stream invertebrates. International Review of Hydrobiology 102:29-37. https://doi.org/10.1002/ iroh.201601856.

Martyniuk, N., B. Modenutti, and E. Balseiro. 2016. Forest structure affects the stoichiometry of periphyton primary producers in mountain streams of northern Patagonia. Ecosystems 19:1225-1239. https://doi.org/10.1007/s10021-0169996-8.

Mauad, M., M. L. Miserendino, M. A. Risso, and J. Massaferro. 2015. Assessing the performance of macroinvertebrate metrics in the Challhuaco-Ñireco System (Northern Patagonia, Argentina). Theringia. Série Zoologia 105:348-358. https://doi.org/10.1590/1678-476620151053348358.

Meissner, K., and T. Muotka. 2006. The role of trout in stream food webs: integrating evidence from field surveys and experiments. Journal of Animal Ecology 75:421-433. https://doi.org/10.1111/j.1365-2656.2006.01063.x.

Mermoz, M., T. Kitzberger, and T. Veblen. 2005. Landscape influences on occurrence and spread of wildfires in patagonian forests and shrublands. Ecology 86:2705-2715. https://doi.org/10.1890/04-1850.

Meyer, J. L., J. B. Wallace, and S. L. Eggert. 1998. Leaf litter as a source of dissolved organic carbon in streams. Ecosystems 1:240-249. https://doi.org/10.1007/s100219900019.

Miserendino, M., M. K. Adriana, B. Cecilia, M. La Ludmila, P. Y. Di Cecilia, P. Gabriela, and B. José. 2016. Ecological status of a Patagonian mountain river: Usefulness of environmental and biotic metrics for rehabilitation assessment. Environ Manage 57:1166-1187. https://doi.org/10.1007/s00267-016-0688-0.

Miserendino, M., C. Brand, and C. Di Prinzio. 2008. Assessing urban impacts on water quality, benthic communities 
and fish in streams of the Andes Mountains, Patagonia (Argentina). Water, air, and soil pollution 194:91-110. https: //doi.org/10.1007/s11270-008-9701-4.

Miserendino, M. L. 2001. Macroinvertebrate assemblages in Andean Patagonian rivers and streams: environmental relationships. Hydrobiologia 444:147-158. https://doi.org/10.1023/A:1017519216789.

Miserendino, M. L., R. Casaux, M. Archangelsky, C. Y. Di Prinzio, C. Brand, and A. M. Kutschker. 2011. Assessing land-use effects on water quality, in-stream habitat, riparian ecosystems and biodiversity in Patagonian northwest streams. Science of The Total Environment 409:612-624. https://doi.org/10.1016/j.scitotenv.2010.10.034.

Miserendino, M. L., and C. I. Masi. 2010. The effects of land use on environmental features and functional organization of macroinvertebrate communities in Patagonian low order streams. Ecological Indicators 10:311-319. https://doi.org/ 10.1016/j.ecolind.2009.06.008.

Modenutti, B., R. Albariño, V. Díaz Villanueva, C. Laspoumaderes, F. Cuassolo, M. S. Souza, M. Bastidas Navarro, C. Trochine, G. Mariluán, and E. Balseiro. 2010. Structure and dynamic of food webs in Andean North Patagonian freshwater systems: organic matter, light and nutrient relationships. Ecología Austral 20:95-114.

Montañez, J. C. 2014. Evaluación de los costos y beneficios de la remoción de salmónidos en un arroyo de montaña del Parque Nacional Nahuel Huapi como herramienta de manejo para la conservación. Licenciatura. Universidad Nacional del Comahue, Centro Regional Universitario Bariloche.

Mykrä, H., J. Heino, and T. Muotka. 2007. Scale-related patterns in the spatial and environmental components of stream macroinvertebrate assemblage variation. Global Ecology and Biogeography 16:149-159. https://doi.org/10.1111/j.14668238.2006.00272.x.

Nuñez, M. N., S. A. Solman, and M. F. Cabré. 2009. Regional climate change experiments over southern South America. II: Climate change scenarios in the late twenty-first century. Climate Dynamics 32:1081-1095. https://doi.org/10.1007/ s00382-008-0449-8.

Paruelo, J. M., A. Beltran, E. Jobbagy, O. E. Sala, and R. A. Golluscio. 1998. The climate of Patagonia: general patterns and controls on biotic. Ecología Austral 8:85-101.

Pascual, M., P. Macchi, J. Urbanski, F. Marcos, C. R. Rossi, M. Novara, and P. Dell'Arciprete. 2002. Evaluating potential effects of exotic freshwater fish from incomplete species presence-absence data. Biological Invasions 4:101-113. https: //doi.org/10.1023/A:1020513525528.

Pauchard, A., R. García, S. Zalba, M. Sarasola, R. Zenni, S. Ziller, and M. A. Nuñez. 2015. 14. Pine Invasions in South America: Reducing Their Ecological Impacts Through Active Management. Pp. 318-342 in Biological Invasions in Changing Ecosystems. Sciendo Migration. https://doi.org/10.1515/9783110438666-020.

Peckarsky, B. L., A. R. McIntosh, M. Álvarez, and J. M. Moslemi. 2013. Nutrient limitation controls the strength of behavioral trophic cascades in high elevation streams. Ecosphere 4:1-17. https://doi.org/10.1890/ES13.00084.1.

Pedrozo, F., S. Chillrud, P. Temporetti, and M. Díaz. 1993. Chemical composition and nutrient limitation in rivers and lakes of northern Patagonian Andes (39.5-42 S; 71 W) (Rep. Argentina). Internationale Vereinigung für theoretische und angewandte Limnologie: Verhandlungen 25:207-214. https://doi.org/10.1080/03680770.1992.11900093.

Pessacg, N., S. Flaherty, L. Brandizi, S. Solman, and M. Pascual. 2015. Getting water right: A case study in water yield modelling based on precipitation data. Science of The Total Environment 537:225-234. https://doi.org/10.1016/ j.scitotenv.2015.07.148.

Petersen, R. C., and K. W. Cummins. 1974. Leaf processing in a woodland stream. Freshwater Biology 4:343-368. https: //doi.org/10.1111/j.1365-2427.1974.tb00103.x.

Petrone, K., I. Buffam, and H. Laudon. 2007. Hydrologic and biotic control of nitrogen export during snowmelt: A combined conservative and reactive tracer approach. Water Resources Research 43(6): W06420. https://doi.org/ 10.1029/2006WR005286.

Piazza, M. V., T. Kitzberger, and E. J. Chaneton. 2018. La deposición de cenizas volcánicas modula la descomposición de hojarasca en bosques de Nothofagus dombeyi del norte de Patagonia. Ecología Austral 28:028-039. https://doi.org/ 10.25260/EA.18.28.1.0.561

Poff, N. L., J. D. Allan, M. B. Bain, J. R. Karr, K. L. Prestegaard, B. D. Richter, R. E. Sparks, and J. C. Stromberg. 1997. The natural flow regime. BioScience 47:769-784. https://doi.org/10.2307/1313099.

Raffaele, E., M. A. Nuñez, and M. A. Relva. 2015. Plantaciones de coníferas exóticas en Patagonia: los riesgos de plantar sin un manejo adecuado. Ecología Austral 25(2):86-157. https://doi.org/10.25260/EA.15.25.2.0.153.

Richardson, J. S., R. B. Bilby, and C. A. Bondar. 2005. Organic matter dynamics in small streams of the pacific northwest. Journal Of The American Water Resources Association 41(4):921-934. https://doi.org/10.1111/j.17521688.2005.tb03777.x.

Rodríguez-Lozano, P., M. Rieradevall, and N. Prat. 2016. Top predator absence enhances leaf breakdown in an intermittent stream. Science of The Total Environment 572:1123-1131. https://doi.org/10.1016/j.scitotenv.2016.08.021.

Rodríguez, P., G. G. Garraza, V. García, M. Granitto, and J. Escobar. 2020. Beaver dam effect on phytoplankton and periphyton composition and hydrology in streams from Tierra del Fuego (Argentina). Hydrobiologia 847:1461-1477. https://doi.org/10.1007/s10750-020-04201-5.

Sarasola, M. M., C. M. Ghersa, V. E. Rusch, and T. M. Schlichter. 2006. Tree conifers invasion in steppe areas and Austrocedus chilensis forests in NW Patagonia. Ecología Austral 16:143-146.

Schäffer, M., C. Winkelmann, C. Hellmann, and J. Benndorf. 2013. Reduced drift activity of two benthic invertebrate species is mediated by infochemicals of benthic fish. Aquatic Ecology 47:99-107. https://doi.org/10.1007/s10452-0139428-1. 
Stubbington, R., and T. Datry. 2013. The macroinvertebrate seedbank promotes community persistence in temporary rivers across climate zones. Freshwater Biology 58:1202-1220. https://doi.org/10.1111/fwb.12121.

Tate, C., and J. L. Meyer. 1983. The influence of hydrologic conditions and successional state on dissolved organic carbon export from forested watersheds. Ecology 64:25-32. https://doi.org/10.2307/1937325.

Temporetti, P. 2006. Efecto a largo plazo de los incendios forestales en la calidad del agua de dos arroyos en la subregión Andino-Patagónica, Argentina. Ecología Austral 16:157-166.

Townsend, C., and M. R. Scarsbrook. 1997. The intermediate disturbance hypothesis, refugia, and biodiversity in streams. Limnology and Oceanography 42:938-949. https://doi.org/10.4319/lo.1997.42.5.0938.

Vannote, R. L., G. W. Minshall, K. W. Cummins, J. R. Sedell, and C. E. Cushing. 1980. The river continuum concept. Canadian Journal of Fisheries and Aquatic Sciences 37:130-137. https://doi.org/10.1139/f80-017.

Veblen, T. T., A. Holz, J. Paritsis, E. Raffaele, T. Kitzberger, and M. Blackhall. 2011. Adapting to global environmental change in Patagonia: What role for disturbance ecology? Austral Ecology 36:891-903. https://doi.org/10.1111/j.14429993.2010.02236.x.

Veblen, T. T., T. Kitzberger, and A. Lara. 1992. Disturbance and forest dynamics along a transect from Andean rain forest to Patagonian shrubland. Journal of Vegetation Science 3:507-520. https://doi.org/10.2307/3235807.

Vigliano, P. H., and M. F. Alonso. 2007. Salmonid introductions in Patagonia: a mixed blessing. Pp. 315-331 in Ecological and genetic implications of aquaculture activities. Springer. https://doi.org/10.1007/978-1-4020-6148-6_17.

Villatarco Vazquez, A. P., D. A. Fernández, and R. J. Albariño. 2019. Drivers of macroinvertebrate metacommunity structure in Tierra del Fuego rivers. Acta Oecologica 97:6-13. https://doi.org/10.1016/j.actao.2019.04.001.

Vitousek, P. M. 1990. Biological invasions and ecosystem processes: towards an integration of population biology and ecosystem studies. Pp. 183-191 in Ecosystem Management. Springer. https://doi.org/10.1007/978-1-4612-4018-1_17.

Von Schiller, D., T. Datry, R. Corti, A. Foulquier, K. Tockner, R. Marcé, G. García-Baquero, I. Odriozola, B. Obrador, and A. Elosegi. 2019. Sediment respiration pulses in intermittent rivers and ephemeral streams. Global Biogeochemical Cycles.

Xenopoulos, M. A., J. A. Downing, M. D. Kumar, S. Menden-Deuer, and M. Voss. 2017. Headwaters to oceans: Ecological and biogeochemical contrasts across the aquatic continuum. Limnology and Oceanography 62:S3-S14. https://doi.org/10.1002/lno.10721.

Ylla, I., I. Sanpera-Calbet, E. Vázquez, A. M. Romaní, I. Muñoz, A. Butturini, and S. Sabater. 2010. Organic matter availability during pre- and post-drought periods in a Mediterranean stream. Hydrobiologia 657:217-232. https: //doi.org/10.1007/s10750-010-0193-z.

Zagarola, J.-P. A., G. Martínez Pastur, M. E. Lopez, and C. B. Anderson. 2017. Assessing the effects of urbanization on streams in Tierra del Fuego. Ecología Austral 27:045-054. https://doi.org/10.25260/EA.17.27.1.0.417. 